\title{
Short tandem repeat polymorphism evolution in humans
}

\author{
F Calafell, A Shuster, WC Speed, JR Kidd and K K Kidd \\ D epartment of G enetics, Yale U niversity School of M edicine, USA
}

\begin{abstract}
Forty-five dinucleotide short tandem repeat polymorphisms were typed in ten large samples of a globally distributed set of populations. Although these markers had been selected for high heterozygosity in European populations, we found them to be sufficiently informative for linkage analysis in nonEuropeans. Heterozygosity, mean number of alleles, and mean number of private alleles followed a common trend: they were highest in the African samples, were somewhat lower in Europeans and East Asians, and were lowest in Amerindians. Genetic distances also reflected this pattern, and distances modelled after the stepwise mutation model yielded trees that were less in agreement with other genetic and archaeological evidence than distances based on differentiation by drift $\left(F_{S T}\right)$. Genetic variation in nonAfricans seems to be a subset of that in Africans, supporting the replacement hypothesis for the origin of modern humans.
\end{abstract}

Keywords: Short tandem repeat polymorphisms; microsatellites; human evolution; genetic distances; replacement hypothesis; multiregional hypothesis

\section{Introduction}

Short tandem repeat polymorphisms (STRPs, also known as microsatellites) present several properties that make them particularly useful in human population genetics: the huge number of STR P loci available ${ }^{1}$ and the relative expediency with which they can be typed allow the gathering of genetic data for a large number of loci in population samples. STR Ps present high heterozygosities and genetic diversities (as measured by $\mathrm{F}_{\mathrm{ST}}$, for instance) comparable to those revealed by blood group and protein polymorphisms (the so-called classical polymorphisms) $)^{2,3}$ or slightly lower. ${ }^{4}$ D ue to their high heterozygosity, STR Ps are expected

Correspondence: Kenneth K K idd, D epartment of G enetics, Yale U niversity School of Medicine, 333 Cedar St, New $\mathrm{H}$ aven, CT 06520-8005, U SA to escape the allele frequency distortions observed at loci that were ascertained on the basis of polymorphism in E uropeans. ${ }^{5}$ M utation in STR Ps appears to follow a stepwise mutation model (SMM), in which mutation events involve the gain or loss of one to a few repeat units. The product of mutation is often an already existing allele; thus, mutation in STRPS violates the basic tenet of the infinite allele model (IAM ), in which every mutation generates a new allele. A strict SM M , in which the gain or loss is limited to one repeat unit per mutation event, produced allele frequency distributions consistent with those observed ${ }^{6}$ but did not account for the observed variability in 10 STRPs in a Sardinian sample. ${ }^{7}$ The average of the mutation rates for STR Ps is several orders of magnitude higher than for single basepair changes in single copy DNA, but there is apparently great variation in rate among loci. ${ }^{8} \mathrm{C}$ hakraborty et $\mathrm{al}^{9}$ analyzed a large number of loci and inferred from 
the repeat-size variance that mutation rate of dinucleotide repeats was higher than that of trinucleotide repeats which, in turn, seemed to mutate faster than tetranucleotide repeats.

Several studies have undertaken the analysis of STR P variation in global sets of populations. B owcock et al $^{10}$ typed 14 populations for 30 STRPs, most of which were dinucleotide repeats that mapped on chromosomes 13 or 15. Eight of the loci were located less than $1 \mathrm{cM}$ away from their nearest neighbour, and linkage disequilibrium was not assessed. O nly small samples (20 chromosomes) of each population were analyzed, and their results showed that individuals tended to cluster by population in a tree based on the proportion of shared alleles. Deka et $\mathrm{al}^{2}$ tested eight dinucleotide repeats in larger samples (100 to 222 chromosomes) of eight populations. Their loci mapped on chromosome 13q; they tested for linkage disequilibrium and did not find it. Reduced levels of heterozygosity were found in Pehuenche (Chile) and D ogrib (Canada) Native A mericans, as well as in $\mathrm{New}$ Guineans. Deka et $\mathrm{al}^{2}$ also observed a significant departure from the allele frequency distributions predicted by a strict SM M. Jorde et al ${ }^{4}$ typed 15 populations for 30 unlinked tetranucleotide STR Ps; except for 140 chromosomes of mixed N orthern E uropean origin, most populations were represented by small numbers of chromosomes (10 to 44). The STRP results were compared to hypervariable segment II mtDNA sequences and to 30 restriction site polymorphisms. They found that, although ST R P data separated A frican populations from the rest, the A frican branches were shallow and, in their interpretation, not supportive of a recent $A$ frican origin of modern humans. L ater, J orde et al ${ }^{11}$ added 27 additional tetranucleotide, two trinucleotide, and one dinucleotide STRPs, and focused on excess A frican heterozygosity, observed by pooling samples within A frica. Finally, Pérez-L ezaun et $\mathrm{al}^{3}$ analyzed 20 unlinked tetranucleotide STRPs in small samples (20 to 30 chromosomes each, except for some of their European samples) of 16 populations. They compared different genetic distances and found that those not based on the SM M produced trees that were in agreement with other genetic and archaeological evidence, whereas SM M-based distances generated tree topologies that were difficult to reconcile with such evidence.

In order to estimate a number of parameters of interest in population genetics, a relatively large number of loci should be typed in samples of reasonable size (eg) ${ }^{12}$ The combined data of a large number of loci may reveal overall patterns that could be masked by the random effects of drift or by specific selection pressures in one or a few loci. Increasing sample size leads to greater statistical power in hypothesis testing, and decreases sampling error. We present data on 45 dinucleotide STRPS, mapping on chromosomes 9, 10, and 11 , at an average distance of $11.3 \mathrm{CM}$ (range: 5-25 CM ). These markers are part of the A BI PRISM ${ }^{\mathrm{TM}}$ Linkage Mapping Sets. Their level of polymorphism and allele frequencies have previously been determined only in a sample consisting of individuals of mostly Northern European origin, with a few individuals of $\mathrm{N}$ ative A merican and A frican ancestry. ${ }^{13}$ We have typed samples from ten specific populations from A frica, E urope, A sia, A ustralo-M elanesia, and the A mericas. Sample sizes, with only one exception, ranged between 80 and 128 chromosomes. The extensive analysis of intra- and interpopulation variability in a large number of loci in large samples allows us to characterize polymorphism in this particular set of markers and to evaluate their usefulness for linkage studies in populations other than E uropeans. The data also illuminated the evolution of human STRPs, in terms of the generation of polymorphism and the characterization of allele distribution. They also support one of the two hypotheses on the origin of anatomically modern Homo sapiens, that is, the replacement model (also known as ' $\mathrm{O}$ ut of $\mathrm{A}$ frica') ${ }^{14}$ in which modern humans have a recent, A frican origin, and replaced archaic Homo, and argue against the multiregional hypothesis, ${ }^{15}$ according to which archaic $\mathrm{H}$ omo evolved into modern humans in parallel throughout the Old World.

\section{Materials and Methods}

\section{Population Samples}

Ten populations, from diverse locations around the world, were typed. These were the M buti Pygmies (Zaire: $2 \mathrm{~N}=78$ ) and the B iaka Pygmies (Central A frican R epublic: $2 N=138$ ) from A frica; the $D$ anes $(2 \mathrm{~N}=124)$ and the $D$ ruze (Northern Israel: $2 \mathrm{~N}=122$ ) from $\mathrm{E}$ urope and the M iddle E ast; Chinese $\mathrm{H}$ an $(2 \mathrm{~N}=98)$, Japanese $(2 \mathrm{~N}=102)$, and Yakut (Siberia: $2 \mathrm{~N}=102$ ) from $\mathrm{E}$ ast A sia; the Nasioi from Bougainville, M elanesia ( $2 \mathrm{~N}=46)$; and $\mathrm{M}$ aya ( $Y$ ucatán, $\mathrm{M}$ exico; $2 \mathrm{~N}=104$ ) and Rondônia Surui (SW A mazonia; $2 \mathrm{~N}=94$ ) from the A mericas. A more extensive description of these samples can be found in, ${ }^{16,17}$ and on the Web page <http://info.med.yale.edu/genetics/kkidd/pops.html $\rangle$. A II of these samples exist as E pstein-Barr virus transformed lymphoblastoid cell lines, which were established under approved human subjects protocols. 
Marker Typing

Forty-five dinucleotide STRPs out of the 51 loci from A BI PRISM ${ }^{\mathrm{TM}}$ L inkage M apping Sets, Panels 13 through 16, were typed; for a list of those loci, see the World Wide Web page 〈http://info.med.yale.edu/genetics/kkidd〉. These markers map to chromosomes 9, 10 and 11 . A II loci were amplified with fluorescently-labeled primer pairs and run in an A BI $373^{\mathrm{TM}}$ DNA Sequencer following manufacturer's protocols with the following modifications: the gel concentration was increased to $8 \%$ acrylamide, the electrophoresis was decreased to $15 \mathrm{~W}$, and the collection time was increased to 7 hours. These changes in gel concentration and wattage increased the consistency of allele-calling between and within populations, by minimizing gel-to-gel variation. R aw data were collected with the 672 GeneScan $^{\text {TM }}$ software and analyzed with $\mathrm{Genotyper}^{\mathrm{TM}}$ software. The decimal base pair sizes output from the Genotyper program were translated into integer values assigned as allele names.

\section{Statistical Analysis}

A llele frequencies were estimated by direct allele counting. Expected heterozygosity was estimated as one minus the sum of squared allele frequencies. ${ }^{18}$ Linkage disequilibrium was tested in contiguous pairs of loci by estimating pairwise haplotype frequencies with the E M -H A PL O program, ${ }^{19}$ from which independence was tested through a log-likelihood ratio $\chi^{2}$ statistic. Heterozygosity, total number of alleles, and number of private alleles were found not to follow normal distributions, and, accordingly, were compared across populations with the appropriate non-parametric tests.

Genetic distances among populations were estimated through $\mathrm{F}_{\mathrm{ST}},{ }^{20,21}$ and three other genetic distances that incorporate the stepwise mutation model: $D_{s w}{ }^{22}(\delta \mu)^{2},{ }^{23}$ and $\mathrm{R}_{\mathrm{ST} .}{ }^{24}$ Neighbor-joining trees ${ }^{25}$ were built from those distances, and the statistical robustness of their nodes were tested through a bootstrap approach: ${ }^{26,27}$ loci were resampled with replacement 1000 times. The LSSearch program ${ }^{28}$ was used to discern whether any tree in the topological vicinity of the neighbor-joining tree was better by least-squares or minimum length criteria. Some of these procedures were performed with the PHY LIP 3.5c package. ${ }^{29}$

\section{Results}

\section{Allele Frequency Distributions and Hardy-Weinberg Equilibrium}

The allele frequencies of 45 STR loci in 10 populations can be retrieved from the World Wide Web page 〈http:/ /info.med.yale.edu/genetics/kkidd $\rangle$. For each locus, we included in the Web page the genotype of one individual from the CEPH families, in order to facilitate meaningful comparisons with datasets produced in the future by other authors in different populations. G lobally, 620 different alleles were detected, with a range of 6 to 29 alleles per locus (mean: 13.8). The observed numbers of heterozygous individuals for every population and locus were tested against $\mathrm{H}$ ardy-Weinberg expectations. O ut of 450 tests, $21(4.67 \%)$ revealed statistically significant discrepancies at a 0.05 significance level, essentially the number expected; after B onferroni correction for multiple tests, only four cases remained significant at a nominal $\alpha=0.05$ significance level. Three of these involved locus D11S934 in the Danes, D ruze, and Nasioi. The large deficiency of heterozygotes could be attributed to a null allele (eg a base-pair variant in the primer complementary sequences) found at varying frequencies in different populations; apparent non-parental transmission consistent with segregation of a null allele was detected in family studies of Europeans (data not shown). The fourth significant heterozygote deficiency involved D 10S591 in the D anes. Null alleles in STR $s$ have been detected in humans ${ }^{30}$ and in bears. ${ }^{31}$ Failure to amplify a ST R P allele could be due to differential amplification if the two allelles have extremely different sizes, or to a mutation in the primer-complementary sequences. Callen et $\mathrm{al}^{30}$ demonstrated an 8-bp deletion in a primer-complementary sequence, and found null alleles in 7 out of 22 dinucleotide loci in chromosome 16. The actual incidence of null alleles is not known; some instances of apparent lack of parental transmission could be, in fact, mutation events.

Even though a mean of 962 chromosomes per locus was typed, the pooled allele distributions of $28(62.2 \%)$ markers were not continuous, with gaps of up to four intermediate allele positions. Within every population, a mean of 31.2 loci $(69.3 \%)$ presented discontinuous allele frequency distributions, with a range from 26 loci in the Japanese to 36 loci in the Surui. At six loci (namely, D 9S161, D 9S273, D 9S290, D 9S164, D 10S192, and D 11S934) we found alleles that differ by one base pair from a perfect two-bp ladder. A t four of six loci, the off-ladder alleles were specific to A frican populations. O ne of those (D 9S164) presented a series of eight A frican-specific alleles interspersed within the two-bp ladder. Complex polymorphism, beyond variation in number of repeats, appears to be a common phenomenon even in dinucleotide STRPs (see ${ }^{2,32}$ for other examples).

\section{Linkage Disequilibrium}

In order to ascertain independence among the loci analyzed, we tested for linkage disequilibrium by estimating two-locus haplotype frequencies for all pairs of adjacent loci, separately in all ten populations. We did not test linkage disequilibrium beyond adjacent loci because the high recombination fractions between loci (eg an average of $20.9 \mathrm{cM}$ for next-to-adjacent pairs of loci) make linkage disequilibrium extremely unlikely. 
From the haplotype frequencies, contingency tables were analyzed through a log-likelihood ratio $\chi^{2}$ statistic. The number of possible haplotypes with two ST R P loci is relatively high, and many will have low expected frequencies; under these conditions, an ordinary $\chi^{2}$ test would perform poorly, and even a log-likelihood ratio is likely to err on the side of rejecting valid null hypotheses. Forty-three pairs of loci were tested in each of the ten populations; after Bonferroni correction, 22 tests remained significant at a nominal $\alpha=0.05$ significance level. Sixteen of the significant tests involved the Surui. Family relations, especially paternal relations, not recorded in ethnographic field notes, could explain this observation. H owever, in all other populations, alleles at each of the loci studied appear to be independent of the alleles present in neighboring loci. This is not an unexpected finding, given the recombination fractions involved (eg an average of $11.3 \mathrm{~cm}$ between adjacent loci) and the presumable absence of extensive selective hitchhiking effects.

\section{Within-population Allele Diversity}

The mean number of different alleles per locus found in A frican populations was $9.67 \pm 0.33(10.31 \pm 0.48$ in the B iaka and $9.02 \pm 0.44$ in the M buti; (Figure 1)), and the number of different alleles per locus was successively lower in Europeans ( $8.90 \pm 0.33)$, E ast A sians $(7.83 \pm 0.25)$, and Native A mericans $(6.12 \pm 0.22)$. Except for the A mericas, these values are higher than those reported by B owcock et al, ${ }^{10}$ which were based on much smaller numbers of individuals per population;

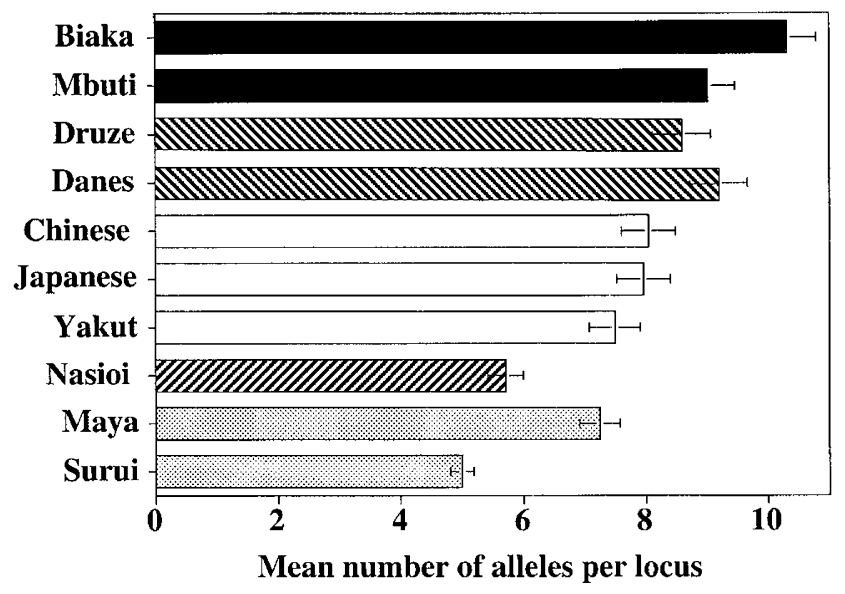

Africa $\mathbb{N}$ Europe $\square$ Asia $\mathscr{Z}$ Australo-Melanesia Americas

Figure 1 M ean number of alleles per locus, estimated from 45 STR P markers. B ars represent standard errors of the mean many of the same individuals were included in the larger samples of those populations in this study. The difference in number of alleles was not statistically significant between $A$ fricans and $E$ uropeans ( $M$ ann Whitney's $U$ test, $p=0.060)$, but it was between E uropeans and $E$ ast $A$ sians $(p=0.010)$, and between East A sians and Native A mericans $(p<0.001)$. A $n$ average of $75.98 \pm 1.96 \%$ of the alleles found worldwide are found in the Biaka Pygmies. In contrast, only $38.65 \pm 1.87 \%$ of the alleles ever seen occur in the A mazonian Surui. A t three loci, all the different alleles ever found in the global sample were present in the Biaka.

A measure of the genetic distinctiveness of a population sample can be obtained from the number of private alleles, ie, those alleles found exclusively in one particular population. Obviously, these are private alleles only with respect to the other nine specific populations in this study. The Biaka had a total of 32 private alleles across 45 loci, the $M$ buti had 18 , and no other population contained more than 9 private alleles (Figure 2). The difference in number of private alleles between the Biaka and the M buti was not statistically significant ( $M$ ann-Whitney's $U, p=0.119$ ), while it was significant at a 0.01 level between the Biaka and any non-A frican population, and, at a 0.05 level, between the $\mathrm{M}$ buti and any non-A frican population (except for the Chinese and the Japanese). Conversely, the Surui had significantly fewer private alleles than all other populations, except for the smaller $\mathrm{N}$ asioi sample. $\mathrm{N}$ ext, we pooled the samples into two groups: A fricans and non-A fricans. A fricans presented a mean of $1.73 \pm 0.30$ A frican-specific alleles per locus, whereas non-A fricans

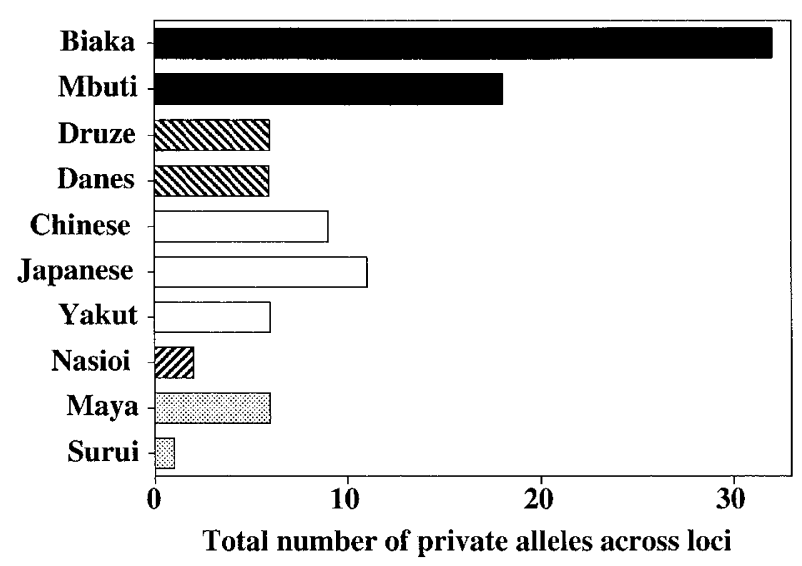

Africa Europe $\square$ Asia $\square$ Australo-Melanesia

Figure 2 Total number of alleles found exclusively in each population, across 45 loci 
Table 1 M ean, standard deviation, range and distribution of expected heterozygosities in 10 populations

\begin{tabular}{|c|c|c|c|c|c|c|c|}
\hline & M ean & s.d. & range & $0-0.25^{a}$ & $0.25-0.5$ & $0.5-0.75$ & $0.75-1$ \\
\hline Biaka & 0.8066 & 0.0087 & $0.5330-0.8976$ & - & - & $9(20 \%)$ & $36(80 \%)$ \\
\hline M buti & 0.7764 & 0.1059 & $0.4175-0.9$ & - & $2(4.4 \%)$ & $13(28.9 \%)$ & $30(66.7 \%)$ \\
\hline Druze & 0.7684 & 0.0077 & $0.5656-0.9185$ & - & - & $16(35.6 \%)$ & $29(64.4 \%)$ \\
\hline Danes & 0.7785 & 0.0072 & $0.5430-0.9007$ & - & - & $15(33.3 \%)$ & $30(66.7 \%)$ \\
\hline Chinese & 0.6745 & 0.1429 & $0.2605-0.8589$ & - & $6(13.3 \%)$ & $22(48.9 \%)$ & $17(37.8 \%)$ \\
\hline Japanese & 0.6678 & 0.1617 & $0.1488-0.8534$ & $2(4.4 \%)$ & $4(8.9 \%)$ & $19(42.2 \%)$ & $20(44.4 \%)$ \\
\hline Y akut & 0.6897 & 0.1327 & $0.2044-0.8597$ & $1(2.2 \%)$ & $3(6.7 \%)$ & $24(53.3 \%)$ & $17(37.8 \%)$ \\
\hline $\mathrm{N}$ asioi & 0.6592 & 0.1324 & $0.2580-0.8367$ & - & $4(8.9 \%)$ & $30(66.7 \%)$ & $11(24.4 \%)$ \\
\hline M aya & 0.6617 & 0.1339 & $0.1665-0.8745$ & $1(2.2 \%)$ & $1(2.2 \%)$ & $29(64.4 \%)$ & $14(31.1 \%)$ \\
\hline Surui & 0.5488 & 0.1905 & $0.0503-0.8103$ & $5(11.1 \%)$ & $8(17.8 \%)$ & $28(62.2 \%)$ & $4(8.9 \%)$ \\
\hline
\end{tabular}

aN umber and percentage of loci with heterozygosities falling in the $0-0.25,0.25-0.5,0.5-0.75$, and $0.75-1$ ranges.

as a whole presented $2.16 \pm 0.27$ alleles specific to nonA fricans. However, the average sample size for nonA fricans was more than three times larger than the average A frican sample size (750.9 vs. 204.7 chromosomes). We corrected for the difference in sample size by taking, for every locus, a random sub-sample with replacement from the non-A frican allele distribution with the sample size of the A fricans, counting how many private alleles were found, and averaging over 10000 sub-samples. Thus, when adjusted for sample size, non-A fricans presented a mean of $1.22 \pm 0.17$ 'private' alleles per locus, that is, $29.5 \%$ less than A fricans. When the same procedure was applied to E uropeans, East A sians, and Native A mericans, the number of private alleles in those continents was always significantly smaller $(p<0.001$, Wilcoxon signed rank test) than the corrected number of alleles found respectively, in non-E uropeans, non-A sians, and non$\mathrm{N}$ ative A mericans. The combined average allele frequency of private alleles was $0.060 \pm 0.011$ in A fricans and $0.044 \pm 0.015$ in non-A fricans ( $p=0.010$, Wilcoxon signed rank test).

In summary, the amount of genetic variability exclusive to $A$ fricans is larger than that of other continents (although a meaningful comparison with the smaller A ustralo-M elanesian sample was not possible), and it is even larger than that of all other continents combined.

Mean and standard deviation of expected heterozygosities are shown in Table 1; most expected heterozygosities fell above 0.5 (Table 1). Depending on the populations, zero to six loci $(0-13.3 \%)$ presented heterozyosities below 0.5 , the only exception being the Surui, with $13(28.9 \%)$ loci below 0.5. Thus, even though they were selected for polymorphism in Europeans, most of the markers in this particular set of STRPS are informative enough to be used in genetic linkage studies in non-E uropean populations. The mean expected heterozygosity (Figure 3) was highest in A frica $(0.792 \pm 0.010)$, it was slightly lower in E urope $(0.773 \pm 0.008)$, and it declines in East A sia $(0.677 \pm 0.013)$, and especially in the Americas $(0.605 \pm 0.018)$. These values are comparable to those found by Jorde et al, ${ }^{11}$ and by Bowcock et al $;{ }^{10}$ sample size does not appear to have a strong effect on the estimation of heterozygosity. A II pairwise differences in heterozygosity between the continental groupings of populations were statistically significant at a 0.05 level (Mann-Whitney's $U$ test).

\section{Allele Size Variance}

Variance can be used to roughly characterize the distribution of the number of repeats in a STRP. At equilibrium, and in the absence of bounds to allele length,

$$
\mathrm{v}=2 \mathrm{~N}_{\mathrm{e}} \mu \sigma_{\mathrm{m}}^{2}
$$

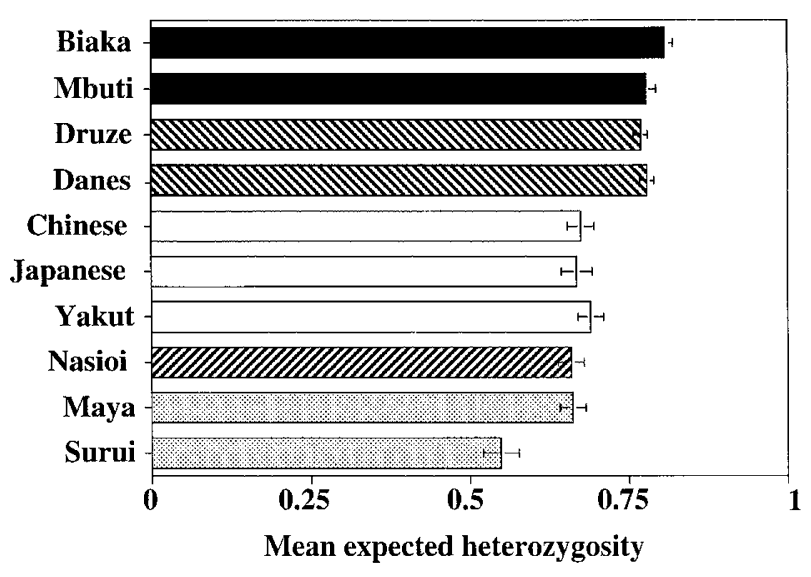

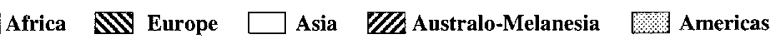

Figure 3 M ean expected heterozygosity in 45 STR P loci. Bars are standard errors of the mean 
where $\mathrm{v}$ is the variance in allele size, $\mathrm{N}_{\mathrm{e}}$ is effective population size, $\mu$ is the mutation rate, and $\sigma_{m}^{2}$ is the variance of the number of repeats gained or lost in every mutation event. ${ }^{24,33-35}$ The average variance in allele size (an indirect measure of repeat number, Figure 4) was $8.11 \pm 0.86$ in A frican populations, $6.48 \pm 0.67$ in Europeans, $6.20 \pm 0.69$ in E ast A sians, and $5.65 \pm 0.82$ in Native A mericans. The distribution of variances across loci was highly skewed to the right, with one locus (D 11S922) presenting a mean variance of 41.61 , more than six times the overall mean variance. A frican populations showed a mode around 5-7; modal values are shifted to lower values in Europeans and East A sians, resulting in lower medians (Table 2), and the distributions of variances across loci in $\mathrm{Native}$ A mericans present peaks at lower values $(<2)$, lower medians, and long right tails (Table 2).

Taking logarithms in E quation (1), a linear model is obtained. ${ }^{9}$ We tested differences in the logarithm of the variance by means of one way analysis of variance

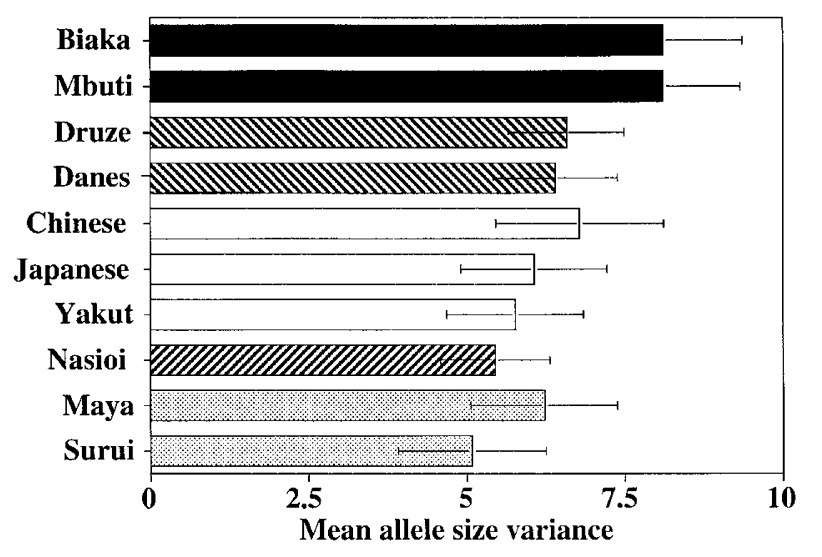

Africa Europe $\square$ Asia $\mathbb{Z 1}$ Australo-Melanesia $\square$ Americas

Figure 4 Allele size variance, averaged across 45 STRPs. A llele size is expressed in number of repeats; bars represent standard errors of the mean

Table 2 M ean, median, and range of the variance in repeat number in 45 STR $P$ loci in 10 populations

\begin{tabular}{lllll}
\hline & M ean & M edian & M inimum & M aximum \\
\hline B iaka & 8.11 & 6.25 & 0.86 & 53.67 \\
M buti & 8.11 & 6.05 & 0.77 & 47.98 \\
D ruze & 6.39 & 4.59 & 0.53 & 43.05 \\
D anes & 6.57 & 5.00 & 1.04 & 37.16 \\
Chinese & 6.78 & 4.15 & 0.26 & 54.97 \\
Japanese & 6.06 & 3.98 & 0.25 & 47.50 \\
Yakut & 5.76 & 4.83 & 0.33 & 46.15 \\
Nasioi & 5.44 & 4.39 & 0.21 & 32.90 \\
M aya & 6.22 & 3.40 & 0.70 & 36.73 \\
Surui & 5.08 & 1.95 & 0.22 & 40.90 \\
\hline
\end{tabular}

(A NOVA), which revealed statistically significant differences across loci $(p<0.001)$. These can be interpreted as differences in the pattern of mutation (the $\mu \sigma_{m}^{2}$ term), as population size is constant across loci. A separate ANOVA showed significant differences by population $(p<0.001)$; thus, different populations could have significantly different present or historical effective population sizes. In a survey of different published datasets, Chakraborty et al ${ }^{9}$ found significant differences for loci within and between motif length classes, but did not find statistically significant differences across populations, probably because Native A merican populations were not included in those datasets. Neither the variance nor its logarithm fit a normal distribution, in violation of the requirements for A NOVA. Thus, we performed a non-parametric test (Kruskal-Wallis' $\mathrm{H}$ ) and found that variances were statistically significantly different $(p<0.001)$ among loci and among populations. However, a non-parametric test does not allow evaluation of fit to a linear model.

In summary, A frican populations present allele distributions with more alleles, more private alleles, and a higher variance than other populations, whereas A merican populations have fewer alleles, fewer private alleles, and repeat number distributions that were less variable on average, but more variable across loci. The implications of these findings will be discussed in detail below.

\section{Genetic Diversity Among Populations}

$M$ ean $\mathrm{F}_{\mathrm{ST}}$ among all populations was $0.0682 \pm 0.0034$, with a range from 0.0279 to 0.1189 . 0 ther global $F_{S T}$ values reported for STRPs are $0.0340,{ }^{4} 0.0859,{ }^{3}$ and $0.1072 .{ }^{2}$ Since $F_{S T}$ measures variances among populations, discrepancies in $\mathrm{F}_{\mathrm{ST}}$ values among populations can be due to the number and choice of populations typed. By continent, the highest interpopulation diversity was found within the Americas $\left(\mathrm{F}_{\mathrm{ST}}=\right.$ $0.0352 \pm 0.0028)$ and A frica $\left(F_{S T}=0.0330 \pm 0.0026\right)$, while the greater interpopulation similarities were found among the E ast $A$ sian $\left(F_{S T}=0.0183 \pm 0.0016\right)$ and $E$ uropean $\left(F_{S T}=0.0146 \pm 0.0011\right)$ samples.

Several genetic distances were computed among the population samples: $\mathrm{F}_{\mathrm{ST}}{ }^{20,21} \mathrm{D}_{\mathrm{SW}}{ }^{22}(\delta \mu)^{2},{ }^{23}$ and $\mathrm{R}_{\mathrm{ST}} \cdot{ }^{24}$ A II distance matrices were positively correlated (Table 3) and all correlation coefficients were statistically significantly different from zero $(p<0.001)$ by the $M$ antel test. ${ }^{36} \mathrm{H}$ owever, most correlation coefficients ranged between 0.6 and 0.75 , which means that only $36 \%$ to $56 \%$ of the variance of one genetic distance can 
be explained by another genetic distance. Therefore, we kept all four distance measures when performing tree analysis.

$\mathrm{G}$ oldstein et $\mathrm{al}^{23}$ showed that, under mutation-drift equilibrium, $(\delta \mu)^{2}$ increases linearly with time, with a slope equal to twice the mutation rate, and, with the dataset published by B owcock et al, ${ }_{1}^{10}$ estimated the age of the A frican vs non-A frican split at 156000 years ago, with a 95\% confidence interval of 75000-287 000 years. The largest $(\delta \mu)^{2}$ value we found was 4.45 between the $M$ buti and the $M$ aya. A pplying the same parameters suggested by $\mathrm{G}$ oldstein et al, ${ }^{23}$ that is, a mutation rate of $5.6 \times 10^{-4}$ and a generation time of 27 years, we obtain a total date (ie, the time needed to accumulate the genetic distance between the $M$ buti and the $M$ aya) for the tree of 107000 years, with a 95\% confidence interval (estimated by 1000 bootstrap iterations as described by $\mathrm{G}$ oldstein et $\mathrm{al}^{23}$ ) of 43500-220000 years, which overlaps with the estimate by $\mathrm{G}$ oldstein et al. ${ }^{23}$ The estimate of divergence time depends linearly on the mutation rate. However, using a more accurate average mutation rate estimate $\left(7.8 \times 10^{-4}, 13\right.$ which is based on typing over 2000 loci (including most of those used in the present study) in large families, we obtained a similar time estimate: 77000 years with a 95\% confidence interval of 31000 to 158000 years ago.

$\mathrm{N}$ eighbor-joining trees were built for all four genetic distances (Figures 5a-d), and the robustness of their branches was tested by bootstrap. The LSSearch program $^{28}$ showed no better (by least squares or minimum length) tree topologies in the vicinity of the neighbor-joining trees. The $F_{S T}$ and $D_{S W}$ trees (Figures $5 a$ and $5 b$ ) showed the same topology: A frican samples clustered on one end, followed by Europeans, $\mathrm{N}$ asioi $M$ elanesians, E ast A sians, and $\mathrm{N}$ ative $\mathrm{A}$ mericans. The same topology was obtained by Cavalli-Sforza et $a^{21}$ with 42 populations and 40 blood groups and protein polymorphisms, totaling 120 independent alleles. For comparison, we can estimate conservatively the range of independent alleles in the 45 STRPs as 94-230 (in the Surui and Biaka, respectively), obtained by counting the number of alleles with frequencies larger than

Table 3 Mantel correlation coefficients ${ }^{36}$ between genetic distances

\begin{tabular}{llll}
\hline & $D_{S W}$ & $(\delta \mu)^{2}$ & $R_{S T}$ \\
\hline$F_{S T}$ & $0.8731^{a}$ & $0.6840^{a}$ & $0.6851^{a}$ \\
$D_{S W}$ & & $0.9231^{a}$ & $0.7200^{a}$ \\
$(\delta \mu)^{2}$ & & & $0.6311^{a}$ \\
\hline
\end{tabular}

ap $<0.001$; significance was estimated from 100000 matrix reshufflings.
0.05 and subtracting one at every locus. Bootstrap supports were high, with only one value slightly below $50 \%$, and similar between the $F_{S T}$ and $D_{S W}$. In particular, branches grouping respectively A fricans, E uropeans, and $\mathrm{N}$ ative $\mathrm{A}$ mericans presented bootstrap supports above $95 \%$. The $(\delta \mu)^{2}$ tree (Figure $5 c$ ) displayed a topology that is harder to reconcile with other genetic and archeological evidence: for instance, the Japanese appear at the base of the branch leading to the A fricans. M oreover, bootstrap supports were lower than for the $F_{S T}$ and $D_{S W}$ trees. Finally, $R_{S T}$ (Figure $5 d$ ) shared the same tree topology with $F_{S T}$ and $D_{S W}$, except for the position of the Melanesian Nasioi, which clustered with the Brazilian Surui.

In terms of branch robustness, previous genetic evidence, and expectations based on current hypotheses on human origins. $F_{S T}$ and $D_{S W}$ performed similarly to each other and better than both $R_{S T}$ and $(\delta \mu)^{2}$. These results agree with those found by PérezLezaun et al, ${ }^{3}$ although their trees were even more discrepant.

\section{Discussion}

A total sample of 962 chromosomes from a global set of 10 populations was typed for 45 dinucleotide STR loci. These loci were originally selected for polymorphism in a sample comprising mostly families of mixed European ancestry ${ }^{13}$ in order to obtain markers suitable for genetic linkage analysis. However, linkage studies are not restricted to E uropean populations (see R eferences 37-39 among many possible examples). Many genetic disorders are confined to specific populations or areas; and isolated populations, which are especially suitable for mapping mutations responsible for genetic disorders, are often of non-E uropean ancestry. In spite of the $E$ uropean bias in their selection, we have shown that almost all of these markers are informative enough for linkage studies in non-E uropeans. E ven in populations such as the Surui that have reduced genetic diversity because of genetic drift, three quarters of these dinucleotide STRPs have heteozygosities above 0.5 , that is, they are more informative than any diallelic marker.

Polymorphism in STR Ps is not restricted to perfect multiples of the repeat unit. In our dataset, six out of 45 loci had alleles interspersed within, but not belonging to, a two-bp ladder. D eka et $\mathrm{al}^{2}$ found such alleles in three out of eight dinucleotide STRPs, and PérezL ezaun et $\mathrm{al}^{40}$ found similar off-ladder alleles at two out 
of twenty tetranucleotide STR Ps. Sequence analysis is needed to determine whether the source of these 'irregular' alleles lies in the flanking regions, ${ }^{41}$ or within the repeat region. It has been shown that STR stretches containing imperfect repeats have a lower mutation rate than perfect repeats, ${ }^{32,42}$ and thus off-ladder alleles could add an additional layer of complexity to STR P mutation patterns. Since they exist at more than $10 \%$ of the loci surveyed, the nature(s) of the mutations that generate them must be a significant factor in STRP evolution.

(a)

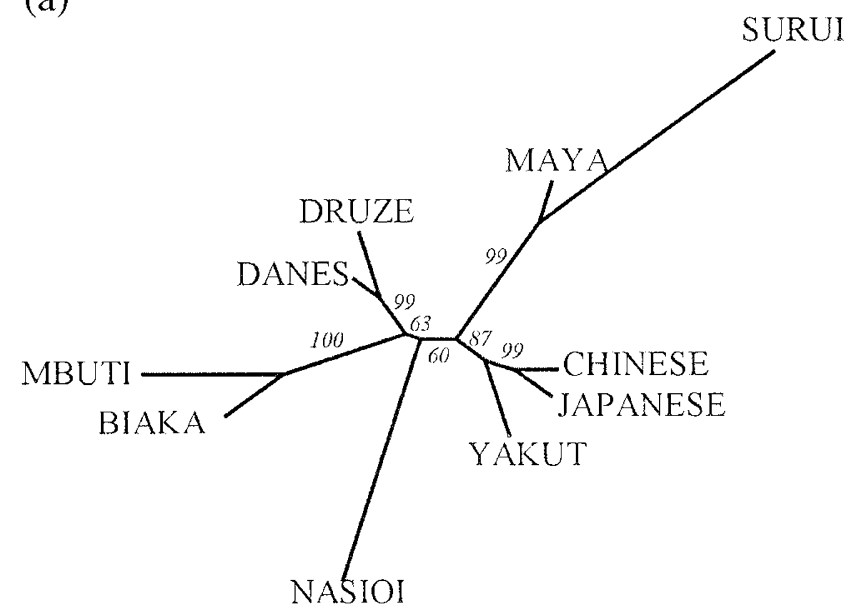

(c)

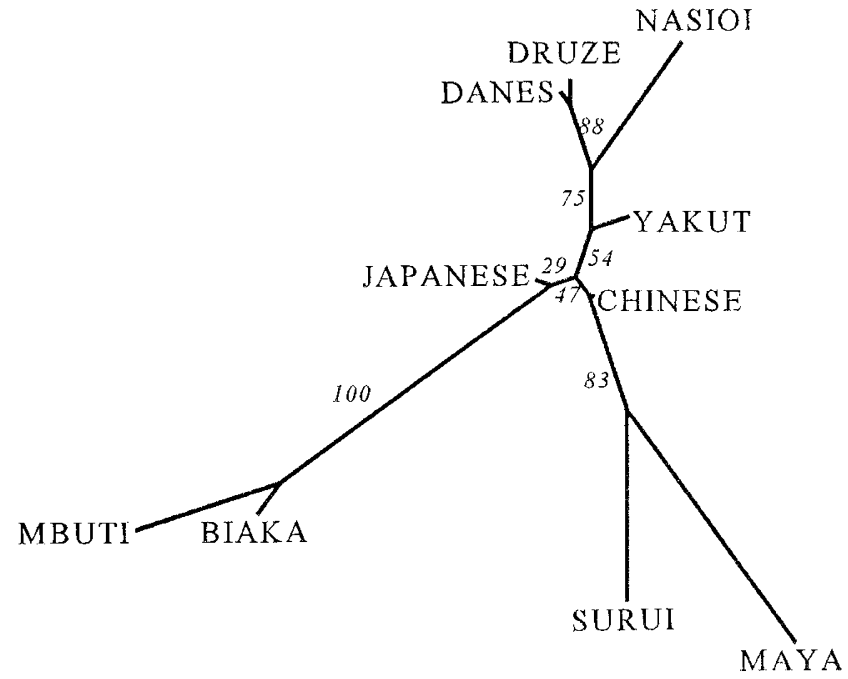

Off-ladder and null alleles are two examples of complex polymorphism at STR loci; base substitutions could also be present in the repeat sequences, ${ }^{43}$ but the methods we used did not allow for their detection. Such complex polymorphism defies attempts to model STR P evolution as the result of an SMM. However, in humans, such a distinction might not be relevant if, as discussed below, drift contributed significantly to shaping STR P allele frequencies.

A fter the discovery of STR Ps and the suggestion that they mutate by adding or subtracting repeat units, there

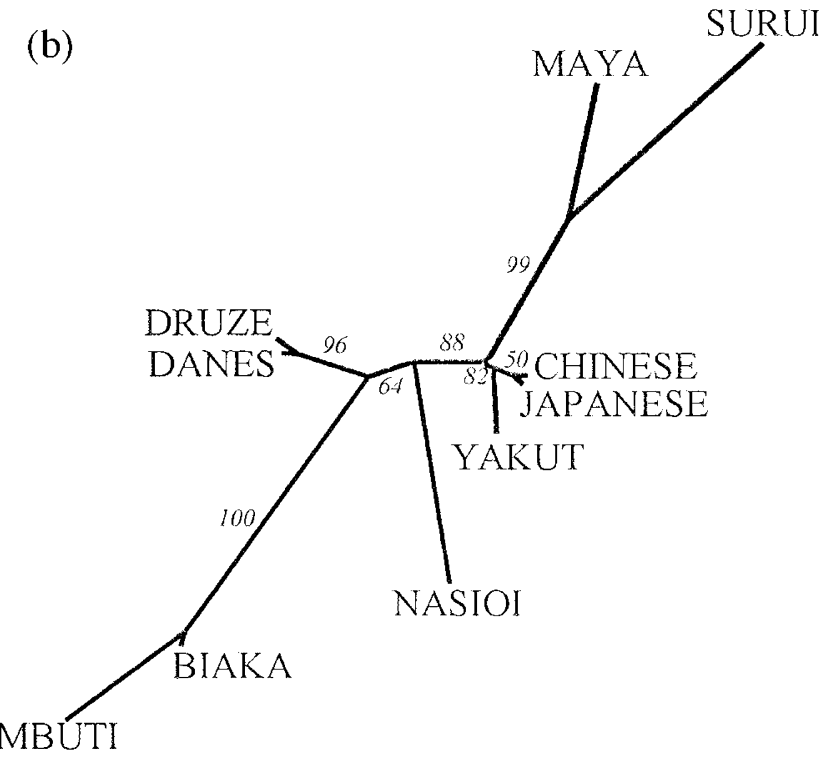

(d) 
has been a notable effort to adapt population genetics parameters that assumed no mutation or an infinite allele model to the stepwise mutation model, and, in particular, several genetic distance measures have been devised specifically for STR Ps. We have used three such STRP-specific genetic distances, and we have found, using the criteria of reasonableness and agreement with archaeological data and other (non-STRP) genetic data, and in agreement with, ${ }^{4}$ that $F_{S T}$ performs as well as, or, in some instances, better than the STR P-specific distance measures. Thus, modeling genetic distances after the SMM does not seem to improve their performance in human STR P analysis. Several reasons can explain this apparent paradox, namely, sampling variance, deviation from the SM M, and genetic drift.

\section{Sampling variance}

$Z$ hivotovski and Feldman ${ }^{44}$ suggested that the variance of $(\delta \mu)^{2}$ grows with the number of loci, and the square of the time elapsed since two populations diverged; N auta and Weissing ${ }^{34}$ noted the high sampling error of $D_{0}$, the mean pairwise difference in number of repeats within a population, a parameter related to $R_{S T}$ and $(\delta \mu)^{2}$. Takezaki and $\mathrm{Nei}^{45}$ simulated the evolution of loci under the SM M in several populations and showed that I A M -appropriate distances, such as Cavalli-Sforza and $E$ dwards' chord distance and $N$ ei et al's $D_{A}$ generally outperformed $D_{s w}$ and $(\delta \mu)^{2}$ in recovering the correct topology of a population tree. They attributed that result to the large sampling variances of $D_{s w}$ and $(\delta \mu)^{2}$. This could also explain why there was greater discrepancy between the trees generated by PérezLezaun et al, ${ }^{4}$ with smaller sample sizes than between ours.

\section{Deviation from the SMM}

This paper and previous reports show that STRP polymorphism is by no means confined to simple variation in repeat number: off-ladder alleles, null alleles, and base substitutions have been repeatedly found. Interruptions of the repeat stretch have been shown to decrease mutation rate, ${ }^{32}$ and longer alleles appear to have higher mutation rates. ${ }^{42}$ In five loci, intermediate alleles were missing from all ten population samples, which could be due to a mutation involving a large jump in the number of repeats occurring in a very deep branch of the coalescent process, ${ }^{6}$ and/or to some mechanical or selective constraint. Thus, departure from a strict SMM could be partly responsible for discontinuous allele distributions. M oreover, allele size can be constrained by upper and lower bounds: ${ }^{34}$ selection against large repeat stretches is seen in the genetic disorders caused by large expansions in some trinucleotide STR PS, although we have not found evidence for such limits in the loci we have analyzed (the variance in repeat number was onethird of that expected under a bound model). The complexity of STRP polymorphism might be oversimplified by a strict SM M, and the distance measures suggested for STR Ps might not capture the complexity of their mutation pattern. However, some attempts have been made to increase the sophistication of the models implemented in STRP-specific genetic distances. Thus, Feldman et $\mathrm{al}^{46}$ devised $D_{L}$, a version of $(\delta \mu)^{2}$ corrected for size boundaries to allele variation. $U$ nfortunately, $D_{L}$ requires that the mutation rate and the maximum number of alleles be known and equal across loci, and we and Chakraborty et $\mathrm{al}^{9}$ have shown that mutation rate is likely to vary across loci.

\section{Genetic Drift}

B eside the deviation factors noted, genetic drift could also have contributed extensively to the creation and maintenance of discontinuous allele distributions. $F_{\text {ST }}$ and other genetic distances were modeled to be linear with separation time between populations and constant population size if differentiation occurred mainly or exclusively by drift. This could the case be for STRP evolution in humans, as argued by Pérez-L ezaun et al. ${ }^{3}$ D rift rather than mutation certainly shaped allele frequencies in genetic polymorphisms such as blood groups and protein electromorphs, ${ }^{21} \mathrm{RFLPs}_{1}{ }^{47}$ and $\mathrm{Alu}$ sequence insertions, ${ }^{48}$ which have a much slower mutation rate. $A$ t the $C D 4$ locus, Tishkoff et $\mathrm{al}^{17}$ showed that, in the CD 4 pentanucleotide, mutation had not restored the allelic diversity in non-A fricans to the level observed in Africans in, according to their interpretation, about 5000 generations. It has been shown ${ }^{18,49}$ that STRP and flanking R FLP alleles at the DRD 2 locus are in strong disequilibrium in multiple populations and that variation in allele frequency at the STR $P$ is accounted for by variation in frequency for the entire haplotype, ie mutation has not restored linkage equilibrium.

Recently, ${ }^{50-52}$ it has been suggested that part of the genetic variation in the $\beta$-globin locus and in the $Y$ chromosome might have been generated in A sia and introduced into A frica by an ancient 'back to A frica' migration. If that were the case for a significant fraction of the genome, we would expect to see a shorter genetic distance between A frica and A sia than between A frica and other continents. This is not the case (Figure 
5a-5d]: in the present analysis of 45 loci, European rather than $E$ ast $A$ sian populations are the closest to A fricans. A significant gene flow from A sia to A frica could have resulted in A frica and $A$ sia sharing a higher number of alleles than A frica and other populations. A cross 45 loci, 22 alleles are shared by A frican and A sian populations and are absent elsewhere; the number of alleles present in A frica and E urope and not elsewhere is considerably larger: 34. Although we cannot rule out a small gene flow from A sia to A frica, large genetic contributions from $A$ sia to $A$ frica seem unlikely.

Several measures of intra-population genetic variability, such as number of alleles, expected heterozygosity, and variance in allele size, showed a consistent pattern in our results. G enetic variability is highest in $A$ frica, intermediate in $E$ urope and $A$ frica, and lowest in the A mericas. The Biaka and the $M$ buti presented intermediate haplotype diversities in the mLD NA control region, ${ }^{53}$ and at the DM PK locus (Tishkoff et al, in preparation) when compared to other A frican populations. The M buti, but not the Biaka, seemed to have an elevated haplotype diversity at the $Y$ chromosome. ${ }^{50}$ Thus, there is no consistent evidence that our choice of A frican populations biased the estimates of intrapopulation diversity. Genetic trees show A fricans at one end and, at successive splits, Europeans, East A sians, and $N$ ative $A$ mericans. A higher heterogeneity in A fricans and/or a genetic tree in which A fricans branch first have been repeatedly shown by different types of nuclear genetic markers: STRPs, 3 ,10,11,17,23 single nucleotide polymorphisms, ${ }^{47}$ minisatellites, ${ }^{54}$ haplotyes, ${ }^{16,17,55}$ and A lu sequences. ${ }^{56}$ This could be explained by a recent $A$ frican origin of anatomically modern humans, which is consistent with the date estimates obtained by us and by $\mathrm{G}$ oldstein et al, ${ }^{23}$ which place an upper limit for the generation of STRP variation at same 220000 years ago. A Iternatively, and as pointed out by R elethford, ${ }^{57}$ a larger population size in A frica could account for a higher A frican heterozygosity and for the apparent greater time depth of A frican genetic diversity. However, such a scenario would probably not be able to accommodate our observation that, on average across 45 nuclear loci, A frican populations present a significantly higher number of private alleles, and, thus, genetic variation outside of A frica tends to be a subset of that of A frica. This has also been observed for the CD 4 locus, ${ }^{17}$ for the DRD 2 locus, ${ }^{16,49}$ and for the $Y$ chromosome. ${ }^{50}$ The relatively high levels of migration needed to synchro- nize evolution in a multi-regional model would also continuously spread the whole A frican variation across the globe, and it seems more likely that one or a few main ' $O$ ut of A frica' migration events spread a subset of the variation generated in A frica.

\section{Acknowledgements}

We appreciate the assistance of Beth Wilson and Tara Coleman in maintaining the lymphoblastoid cell lines. The A pplied Biosystems Division of Perkin-Elmer provided L inkage M apping Sets and other materials. Supported in part by U SPHS grants M H 39329 and MH 50390 (to KKK), and NSF grant SBR-940834 (to JRK). FC is the recipient of a postdoctoral fellowship from CIRIT, A utonomous Government of Catalonia.

\section{References}

$1 \mathrm{D}$ ib $\mathrm{C}$ et al: A comprehensive genetic map of the human genome based on 5624 microsatellites. Nature 1996; 380: 152-154.

2 Deka $R$ et al: Population genetics of dinucleotide $(\mathrm{dC}-\mathrm{dA}) \mathrm{n}$ (dG-dT)n polymorphisms in World populations. A m J H um G enet 1995; 56: 461-474.

3 Pérez-Lezaun et al: Microsatellite variation and the differentiation of modern humans. H um G enet 1997; 99: 1-7.

4 Jorde L B et al: O rigins and affinities of modern humans: $a$ comparison of mitochondrial and nuclear genetic data. A m J H um G enet 1995; 57: 523-538.

5 R ogers A R, Jorde L B: A scertainment bias in estimates of average heterozygosity. Am J Hum Genet 1996; 58: 1033-1041.

6 Valdes A M, Slatkin M, Freimer NB : A llele frequencies at microsatellite loci: the stepwise mutation model revisited. G enetics 1993; 133: 737-749.

$7 \mathrm{Di}$ Rienzo A et al: Mutational processes of simple sequence repeat loci in human populations. Proc Natl A cad Sci USA 1994; 91: 3166-3170.

8 Weber J, Wong C: Mutation of human short tandem repeats. H um M olec G en 1993; 2: 1123-1128.

9 Chakraborty $\mathrm{R}$ et al: Relative mutation rates at di-, triand tetranucleotide microsatellite loci. P roc N atl A cad Sci USA 1997; 94: 1041-1046.

10 B owcock A M et al: $\mathrm{H}$ igh resolution of human evolutionary trees with polymorphic microsatellites. Nature 1994; 368: 455-457.

11 Jorde LB et al: Microsatellite diversity and the demographic history of modern humans. Proc N atl Acad Sci USA 1997; 94: 3100-3103.

12 A stolfi P, Kidd KK, Cavalli-Sforza LL: A comparison of methods for reconstructing evolutionary trees. Syst Z ool 1981; 30: 156-169.

13 G yapay G et al: The 1993-1994 G énéthon human genetic linkage map. Nat G enet 1994 7: 246-339.

14 Stringer $C B, A$ ndrews $P: G$ enetic and fossil evidence for the origin of modern humans. Science 1988; 239: 1263-1268. 
15 Wolpoff $\mathrm{MH}$ : Multiregional evolution: the fossil alternative to $E$ den. In: M ellar $P$, Stringer $C$ (eds.)The $H$ uman Revolution: B ehavioural and B iological Perspectives on the O rigins of Modern Humans, Princeton U niversity Press, Princeton, NJ, 1989, pp62-108.

16 Castiglione $C M$ et al: Evolution of haplotypes at the DRD 2 locus. A m J H um G enet 1995; 57: 1445-1456.

17 Tishkoff SA et al: Global patterns of linkage disequilibrium at the CD 4 locus and modern human origins. Science 1996; 271: 1380-1387.

18 Weir BS: G enetic data analysis II. Sinauer A ssociates, Sunderland, M A, 1996.

$19 \mathrm{H}$ awley $\mathrm{ME}, \mathrm{K}$ id KK: HA PLO : a program using the $\mathrm{EM}$ algorithm to estimate the frequencies of multi-site haplotyes. J H ered 1995; 86: 409-411.

20 Wright S: The genetical structure of populations. Ann E ugenet 1951; 15: 323-354.

21 Cavalli-Sforza LL, Menozzi P, Piazza A: History and geography of human genes. Princeton U niversity Press, Princeton, NJ, 1994.

22 Shriver MD et al: A novel measure of genetic distance for highly polymorphic tandem repeat loci. Mol Biol Evol 1995; 12: 914-920.

23 Goldstein DB, Ruiz-Linares A, Cavalli-Sforza LL, Feldman $M W$ :G enetic absolute dating based on microsatellites and the origin of modern humans. Proc Natl A cad Sci USA 1995; 92: 6723-6727.

24 Slatkin M: A measure of population subdivision based on microsatellite allele frequencies. Genetics 1995; 139: 457-462.

25 Saitou N, Nei M: The neighbor-joining method: a new method for reconstructing phylogenetic trees. $\mathrm{Mol} \mathrm{Biol}$ Evol 1987; 4: 406-425.

26 Efron B: The jackknife, the bootstrap, and other resampling plans. Society for Industrial and A pplied Mathematics, Philadelphia, PA, 1982.

27 Felsenstein J: Confidence limits on phylogenies: an approach using the bootstrap. Evolution 1985; 39: 783-791.

28 Kidd KK, Sgaramella-Z onta LA : Phylogenetic analysis: concepts and methods. Am J Hum Genet 1971; 23: 235-252.

29 Felsenstein J: PHY LIP - Phylogeny Inference Package (Version 3.2). Cladistics 1989; 5: 164-166.

30 Callen D F et al: Incidence and origin of 'null' alleles in the (A C ) n microsatellite markers. A m J H um G enet 1993; 52: 922-927.

31 Paetkau D, Strobeck C: The molecular basis and evolutionary history of a microsatellite null allele in bears. $\mathrm{M} \mathrm{ol}$ E col 1995; 4: 519-520.

32 J in L et al: Mutation rate varies among alleles at a microsatellite locus: phylogenetic evidence. Proc Natl A cad Sci USA 1996; 93: 15285-15288.

33 Moran PAP: Wandering distributions and the electrophoretic profile. Theor pop biol 1975; 8: 318-330.

34 Nauta MJ, Weissing FJ: Constraints on allele size at microsatellite loci: implications for genetic differentiation. G enetics 1996; 143: 1021-1032.

35 K immel M, Chakraborty R, Stivers D N, D eka R: Dynamics of repeat polymorphisms under a forward-backward mutation model: within and between variability at microsatellite loci. G enetics 1996; 143: 549-555.
$36 \mathrm{M}$ antel $\mathrm{N}$ : The detection of disease clustering and a generalized regression approach. Cancer Res 1967; 27: 209-220.

37 Takiyama $Y$ et al: The gene for $M$ achado-J oseph disease maps to human chromosome 14q. Nat Gen 1993; 4: 300-304.

$38 \mathrm{Yu}$ CE et al: Positional cloning of the Werner's syndrome gene. Science 1996; 272: 258-262.

39 Norman RA et al: A bsence of linkage of obesity and energy metabolism to markers flanking homologues or rodent obesity genes in Pima Indians. D iabetes 1996; 45: 1229-1232.

40 Pérez-Lezaun A et al: A llele frequencies for 20 microsatellite loci in a worldwide population survey. Hum H ered 1997; 47: 189-196.

41 G rimaldi M C, Crouau-R oy B: M icrosatellite allelic homoplasy due to variable flanking sequences. J M ol E vol 1997; 44 336-340.

42 M urray $A$ et al: The role of size, sequence and haplotype in the stability of FRAXA and FRAXE alleles during transmission. H um M olec G en 1997; 6: 173-184.

43 Pérez-L ezaun A : I dentification of a base pair substitution at the tetranucleotide tandem repeat locus DHFRP2(AAAC)n using non-denaturing gel electrophoresis. Int J L eg Med 1996; 109: 159-160.

44 Zhivotovsky LA, Feldman M W: M icrosatellite variability and genetic distances. Proc Natl A cad Sci USA 1995; 92: 11549-11552.

45 Takezaki N, Nei M : Genetic distances and reconstruction of phylogenetic trees from microsatellite DNA. G enetics 1996; 144: 389-399.

46 Feldman M W, Bergman A, Pollock DD, Goldstein D B : Microsatellite genetic distances with range constraints: analytic description and problems of estimation. G enetics 1997; 145: 207-216.

47 Bowcock $A M$ et al: D rift, admixture and selection in human evolution: A study with DNA polymorphisms. Proc Natl A cad Sci 1991; 88: 839-843.

48 Batzer MA et al: A frican origin of human-specific polymorphic A lu insertions. Proc Natl A cad Sci USA 1994; 91 12288-12292.

$49 \mathrm{~K}$ idd KK et al: DR 2 haplotypes containing the Taql A 1 allele: implications for alcoholism research. A Icohol Clin Exp Res 1996; 20: 697-705.

$50 \mathrm{H}$ ammer M F et al: The geographic distribution of human Y chromosome variation. G enetics 1997; 145: 785-805.

51 A Itheide TK, $\mathrm{H}$ ammer M F : E vidence for a possible A sian origin of YA P + Y chromosomes. A m J H um G enet 1997; 61: 462-466.

$52 \mathrm{H}$ arding $\mathrm{R} M$ et al: A rchaic $A$ frican and $A$ sian lineages in the genetic ancestry of modern humans. A $\mathrm{m} J \mathrm{H}$ um $\mathrm{G}$ enet 1997; 60: 772-789.

53 Watson $E$ et al: $m$ tD NA sequence diversity in A frica. A m J H um G enet 1996; 59: 437-444.

54 A rmour JA et al: M inisatellite diversity supports a recent A frican origin for modern humans. $N$ at $G$ en 1996; 13: 154- 160.

$55 \mathrm{~K}$ idd $\mathrm{KK}, \mathrm{Kidd} \mathrm{JR}$ : A nuclear perspective on human evolution. In: Boyce AJ, Mascie-Taylor CGN (eds.) Molecular Biology and Human Diversity Cambridge U niversity Press, Cambridge, U K, 1996, pp242-264. 
$56 \mathrm{~K}$ night $\mathrm{A}$ et al: DNA sequences of the A lu elements indicate a recent replacement of the human autosomal genetic complement. Proc Natl A cad Sci USA 1996; 93: 4360-4364.
57 R elethford J: Genetics and modern human origins. E vol A nthropol 1995; 4: 53-63. 